\title{
A note on perfect matchings in uniform hypergraphs
}

\author{
Andrew Treglown* \\ School of Mathematics \\ University of Birmingham \\ Birmingham, UK \\ a.c.treglown@bham.ac.uk
}

\author{
Yi Zhao ${ }^{\dagger}$ \\ Department of Mathematics and Statistics \\ Georgia State University \\ Atlanta, USA \\ yzhao6@gsu.edu
}

Submitted: Jul 14, 2015; Accepted: Jan 13, 2016; Published: Jan 22, 2016

Mathematics Subject Classifications: 05C70, 05C65

\begin{abstract}
We determine the exact minimum $\ell$-degree threshold for perfect matchings in $k$ uniform hypergraphs when the corresponding threshold for perfect fractional matchings is significantly less than $\frac{1}{2}\left(\begin{array}{c}n \\ k-\ell\end{array}\right)$. This extends our previous results that determine the minimum $\ell$-degree thresholds for perfect matchings in $k$-uniform hypergraphs for all $\ell \geqslant k / 2$ and provides two new (exact) thresholds: $(k, \ell)=(5,2)$ and $(7,3)$.
\end{abstract}

Keywords: perfect matchings, hypergraphs, absorbing method

\section{Introduction}

A perfect matching in a hypergraph $H$ is a collection of vertex-disjoint edges of $H$ which cover the vertex set $V(H)$ of $H$. Given a $k$-uniform hypergraph $H$ with an $\ell$-element vertex set $S$ (where $0 \leqslant \ell \leqslant k-1$ ) we define $d_{H}(S)$ to be the number of edges containing $S$. The minimum $\ell$-degree $\delta_{\ell}(H)$ of $H$ is the minimum of $d_{H}(S)$ over all $\ell$-element sets of vertices in $H$. In recent years the problem of determining the minimum $\ell$-degree threshold that ensures a perfect matching in a $k$-uniform hypergraph has received much attention (see e.g. [9, 17, 15, 18, 5, 14, 12, 7, 8, 1, 19, 2, 20, 6, 11, 3]). See [16] for a survey on matchings (and Hamilton cycles) in hypergraphs.

Suppose that $\ell, k, n \in \mathbb{N}$ such that $k \geqslant 3, \ell \leqslant k-1$ and $k$ divides $n$. Let $m_{\ell}(k, n)$ denote the smallest integer $m$ such that every $k$-uniform hypergraph $H$ on $n$ vertices with $\delta_{\ell}(H) \geqslant m$ contains a perfect matching. The conjectured value of $m_{\ell}(k, n)$ comes from two types of constructions. The first type of constructions are referred to as divisibility

*Supported by EPSRC grant EP/M016641/1.

†Supported by NSF grant DMS-1400073. 
barriers. Given a set $V$ of $n$ vertices with a partition $A, B$, let $E_{\text {odd }}(A, B)\left(E_{\text {even }}(A, B)\right)$ denote the family of all $k$-element subsets of $V$ that intersect $A$ in an odd (even) number of vertices. Define $\mathcal{B}_{n, k}(A, B)$ to be the $k$-uniform hypergraph with vertex set $V$ and edge set $E_{\text {odd }}(A, B)$. Note that the complement $\overline{\mathcal{B}}_{n, k}(A, B)$ of $\mathcal{B}_{n, k}(A, B)$ has edge set $E_{\text {even }}(A, B)$. Define $\mathcal{H}_{\text {ext }}(n, k)$ to be the collection of the following hypergraphs. First, $\mathcal{H}_{\text {ext }}(n, k)$ contains all hypergraphs $\overline{\mathcal{B}}_{n, k}(A, B)$ where $|A|$ is odd. Second, if $n / k$ is odd then $\mathcal{H}_{\text {ext }}(n, k)$ also contains all hypergraphs $\mathcal{B}_{n, k}(A, B)$ where $|A|$ is even; if $n / k$ is even then $\mathcal{H}_{\text {ext }}(n, k)$ also contains all hypergraphs $\mathcal{B}_{n, k}(A, B)$ where $|A|$ is odd. It is easy to see that no hypergraph in $\mathcal{H}_{\text {ext }}(n, k)$ contains a perfect matching. Define $\delta(n, k, \ell)$ to be the maximum of the minimum $\ell$-degrees among all the hypergraphs in $\mathcal{H}_{\text {ext }}(n, k)$. Note that $\delta(n, k, \ell)=(1 / 2+o(1))\left(\begin{array}{l}n-\ell \\ k-\ell\end{array}\right)$ but the general formula of $\delta(n, k, \ell)$ is unknown (see more discussion in [19]).

The other type of extremal constructions are referred to as space barriers. Let $H^{*}(n, k)$ be the $k$-uniform hypergraph on $n$ vertices whose vertex set is partitioned into two vertex classes $A$ and $B$ of sizes $n / k-1$ and $(1-1 / k) n+1$ respectively and whose edge set consists precisely of all those edges with at least one endpoint in $A$. Then $H^{*}(n, k)$ does not have a perfect matching and $\delta_{\ell}\left(H^{*}(n, k)\right)=\left(\begin{array}{l}n-\ell \\ k-\ell\end{array}\right)-\left(\begin{array}{c}(1-1 / k) n-\ell+1 \\ k-\ell\end{array}\right) \approx\left(1-\left(\frac{k-1}{k}\right)^{k-\ell}\right)\left(\begin{array}{l}n-\ell \\ k-\ell\end{array}\right)$.

An asymptotic version of the following conjecture appeared in $[5,10]$ and the minimum vertex degree version was stated in [12].

Conjecture 1. Let $k, \ell \in \mathbb{N}$ such that $\ell \leqslant k-1$. Then for sufficiently large $n \in k \mathbb{N}$,

$$
m_{\ell}(k, n)=\max \left\{\delta(n, k, \ell),\left(\begin{array}{l}
n-\ell \\
k-\ell
\end{array}\right)-\left(\begin{array}{c}
(1-1 / k) n-\ell+1 \\
k-\ell
\end{array}\right)\right\}+1 .
$$

Note that for all $1 \leqslant \ell \leqslant k-1$,

$$
\left(\frac{k-1}{k}\right)^{k-\ell}<\left(\frac{1}{e}\right)^{1-\frac{\ell}{k}} \text { and } 1-\left(\frac{k-1}{k}\right)^{k \ln 2} \rightarrow \frac{1}{2} \quad \text { as } \quad k \rightarrow \infty,
$$

where $\ln$ denotes the natural logarithm function. Thus, for $1 \ll k \ll n$, if $\ell$ is significantly bigger than $(1-\ln 2) k \approx 0.307 k$ then $\delta(n, k, \ell)>\left(\begin{array}{l}n-\ell \\ k-\ell\end{array}\right)-\left(\begin{array}{c}(1-1 / k) n-\ell+1 \\ k-\ell\end{array}\right)$. On the other hand, if $\ell$ is smaller than $(1-\ln 2) k$ then $\delta(n, k, \ell)<\left(\begin{array}{l}n-\ell \\ k-\ell\end{array}\right)-\left(\begin{array}{c}(1-1 / k) n-\ell+1 \\ k-\ell\end{array}\right)$ for sufficiently large $n$.

Conjecture 1 has been proven in a number of special cases. Indeed, Rödl, Ruciński and Szemerédi [18] proved the conjecture for $\ell=k-1$. The authors [19, 20] generalized this result by showing $m_{\ell}(k, n)=\delta(n, k, \ell)+1$ for all $k / 2 \leqslant \ell \leqslant k-1$ (independently Czygrinow and Kamat [2] proved this for $(k, \ell)=(4,2))$. In the case when $(k, \ell)=(3,1)$, Conjecture 1 was confirmed by Kühn, Osthus and Treglown [12] and independently Khan [7]. Khan [8] also resolved the case when $(k, \ell)=(4,1)$. Alon, Frankl, Huang, Rödl, Ruciński and Sudakov [1] determined $m_{\ell}(k, n)$ asymptotically in the case when $(k, \ell)=(5,1),(5,2)$, $(6,2)$, and $(7,3)$. Other than these results, no other asymptotic or exact results are known (the best known general bounds are due to Kühn, Osthus and Townsend [11]).

A connection between $m_{\ell}(k, n)$ and the minimum $\ell$-degree that forces a perfect fractional matching was discovered in [1]. Let $H$ be a $k$-uniform hypergraph on $n$ vertices. A 
fractional matching in $H$ is a function $w: E(H) \rightarrow[0,1]$ such that for each $v \in V(H)$ we have that $\sum_{e \ni v} w(e) \leqslant 1$. Then $\sum_{e \in E(H)} w(e)$ is the size of $w$. If the size of the largest fractional matching $w$ in $H$ is $n / k$ then we say that $w$ is a perfect fractional matching. Given $k, \ell \in \mathbb{N}$ such that $\ell \leqslant k-1$, define $c_{k, \ell}^{*}$ to be the smallest number $c$ such that every $k$-uniform hypergraph $H$ on $n$ vertices with $\delta_{\ell}(H) \geqslant(c+o(1))\left(\begin{array}{l}n-\ell \\ k-\ell\end{array}\right)$ contains a perfect fractional matching. It is easy to see that the hypergraph $H^{*}(n, k)$ defined earlier contains no perfect fractional matching. Thus $c_{k, \ell}^{*} \geqslant 1-\left(\frac{k-1}{k}\right)^{k-\ell}$. Alon et al. [1, Theorem 1.1] showed that for fixed $k, \ell$, as $n \in k \mathbb{N}$ tends to infinity,

$$
m_{\ell}(k, n)=\left(\max \left\{\frac{1}{2}, c_{k, \ell}^{*}\right\}+o(1)\right)\left(\begin{array}{l}
n-\ell \\
k-\ell
\end{array}\right) .
$$

Furthermore, in [1] the authors conjectured that $c_{k, \ell}^{*}=1-\left(\frac{k-1}{k}\right)^{k-\ell}$ and confirmed this for $\ell \geqslant k-4$. Together with (1), this gives the aforementioned (asymptotic) results on $m_{\ell}(k, n)$ for $(k, \ell)=(5,1),(5,2),(6,2)$ and $(7,3)$.

In this note we prove the following refinement of $(1)$.

Theorem 2. Fix $k, \ell \in \mathbb{N}$ with $\ell \leqslant k-1$ and let $n \in k \mathbb{N}$. Then

$$
m_{\ell}(k, n)=\max \left\{\delta(n, k, \ell)+1,\left(c_{k, \ell}^{*}+o(1)\right)\left(\begin{array}{l}
n-\ell \\
k-\ell
\end{array}\right)\right\} .
$$

Although it looks like a small improvement, Theorem 2 enables us to determine $m_{\ell}(k, n)$ exactly whenever $c_{k, \ell}^{*}<1 / 2$. A recent result of Kühn, Osthus and Townsend [11, Theorem 1.9] showed that

$$
c_{k, \ell}^{*} \leqslant \frac{k-\ell}{k}-\frac{k-\ell-1}{k^{k-\ell}}
$$

for all $\ell \leqslant k-2$. Together with $c_{k, k-1}^{*}=1 / k$ (see [17]), this implies that $c_{k, \ell}^{*}<1 / 2$ for all $k / 2 \leqslant \ell \leqslant k-1$. Consequently Theorem 2 implies the aforementioned results of [19, 20]: $m_{\ell}(k, n)=\delta(n, k, \ell)+1$ for all $k / 2 \leqslant \ell \leqslant k-1$.

Furthermore, in [1] it was shown that $c_{5,2}^{*}=61 / 125<1 / 2$ and $c_{7,3}^{*}=1105 / 2401<1 / 2$. Therefore an immediate consequence of Theorem 2 is the following corollary.

Corollary 3. Suppose that $(k, \ell)=(5,2)$ or $(7,3)$. Then $m_{\ell}(k, n)=\delta(n, k, \ell)+1$ for sufficiently large $n$.

After this paper was submitted, Han [4] showed that $c_{k, \ell}^{*}<1 / 2$ in the case when $0.42 k \leqslant \ell<k / 2$ or $(k, \ell)=(12,5),(17,7)$. Thus, together with Theorem 2 this resolves Conjecture 1 in these cases.

Let us highlight the ideas behind the proof of Theorem 2. It is informative to first recall the proof of (1), which is an application of the absorbing method. The authors of [1] first applied a lemma of Hàn, Person and Schacht [5, Lemma 2.4], which states that every $k$-uniform hypergraph $H$ with $\delta_{\ell}(H) \geqslant(1 / 2+o(1))\left(\begin{array}{c}n \\ k-\ell\end{array}\right)$ contains a small matching $M$ that can absorb any vertex set $W \subset V(H) \backslash V(M)$ of size much smaller than $M$ (that is, there is matching in $H$ on $V(M) \cup W$ exactly). Next they found an almost perfect matching in 
$H[V(H) \backslash V(M)]$ by first finding a fractional matching and then converting it to an integer matching. This immediately provides the desired perfect matching of $H$ because $M$ can absorb all uncovered vertices in $V(H) \backslash V(M)$. In order to prove Theorem 2, we prove a result stronger than [5, Lemma 2.4], Theorem 5, which implies that every $k$-uniform hypergraph $H$ with $\delta_{\ell}(H) \geqslant(1 / 2-o(1))\left(\begin{array}{c}n \\ k-\ell\end{array}\right)$ either contains the aforementioned $M$ or looks like a hypergraph in $\mathcal{H}_{\text {ext }}(n, k)$. If $H$ contains $M$, then we proceed as in [1] (except that we apply a lemma from [11] when converting a fractional matching to an integer matching); if $H$ looks like a hypergraph in $\mathcal{H}_{\text {ext }}(n, k)$ and $\delta_{\ell}(H) \geqslant \delta(n, k, \ell)+1$, then we obtain a perfect matching by applying [19, Theorem 4.1].

Theorem 5 is the main contribution of this note - it is stronger than two absorbing theorems in our previous papers [19, Theorem 3.1] and [20, Theorem 3.1], in which we assume that $\ell \geqslant k / 2$. The proof of Theorem 5 is actually shorter than those of the two previous absorbing theorems because 1) we use a different absorbing structure which allows us to apply a lemma from [13]; 2) when proving Lemma 10, we avoid using auxiliary hypergraphs and obtain the structure of $H$ by considering the neighborhoods of the vertices of $H$ directly.

Notation: Given a set $X$ and $r \in \mathbb{N}$, we write $\left(\begin{array}{l}X \\ r\end{array}\right)$ for the set of all $r$-element subsets of $X$. Let $H$ be a $k$-uniform hypergraph. We write $V(H)$ for the vertex set and $E(H)$ for the edge set of $H$. Define $e(H):=|E(H)|$. Given $v \in V(H)$, we write $N_{H}(v)$ to denote the neighborhood of $v$, that is, the family of those $(k-1)$-subsets of $V(H)$ which, together with $v$, form an edge in $H$. Given $X \subseteq V(H)$, we write $H[X]$ for the subhypergraph of $H$ induced by $X$, namely, $H[X]:=\left(X, E(H) \cap\left(\begin{array}{l}X \\ k\end{array}\right)\right)$. We denote the complement of $H$ by $\bar{H}$. That is, $\bar{H}:=\left(V(H),\left(\begin{array}{c}V(H) \\ k\end{array}\right) \backslash E(H)\right)$. Suppose that $n, k \in \mathbb{N}$. When $|A|=\lfloor n / 2\rfloor$ and $|B|=\lceil n / 2\rceil$, we define $\mathcal{B}_{n, k}:=\mathcal{B}_{n, k}(A, B)$ and $\overline{\mathcal{B}}_{n, k}:=\overline{\mathcal{B}}_{n, k}(A, B)$.

We will often write $0<a_{1} \ll a_{2} \ll a_{3}$ to mean that we can choose the constants $a_{1}, a_{2}, a_{3}$ from right to left. More precisely, there are increasing functions $f$ and $g$ such that, given $a_{3}$, whenever we choose some $a_{2} \leqslant f\left(a_{3}\right)$ and $a_{1} \leqslant g\left(a_{2}\right)$, all calculations needed in our proof are valid. Hierarchies with more constants are defined in the obvious way.

\section{Proof of Theorem 2}

The lower bound for $m_{\ell}(k, n)$ in Theorem 2 follows from the definitions of $\delta(n, k, \ell)$ and $c_{k, \ell}^{*}$ immediately. The following (more general) result provides the desired upper bound for $m_{\ell}(k, n)$.

Theorem 4. Given any $\theta>0, k, \ell, \ell^{\prime} \in \mathbb{N}$ where $1 \leqslant \ell, \ell^{\prime} \leqslant k-1$ there is an $n_{0} \in \mathbb{N}$ such that the following holds. Let $n \geqslant n_{0}$ where $k$ divides $n$. If $H$ is a $k$-uniform hypergraph on $n$ vertices with

$$
\delta_{\ell}(H)>\delta(n, k, \ell) \quad \text { and } \quad \delta_{\ell^{\prime}}(H)>\left(c_{k, \ell^{\prime}}^{*}+\theta\right)\left(\begin{array}{c}
n-\ell^{\prime} \\
k-\ell^{\prime}
\end{array}\right)
$$

then $H$ contains a perfect matching. 
The proof of Theorem 4 splits into extremal and non-extremal cases, the former case being when $H$ looks like an element of $\mathcal{H}_{\mathrm{e} x t}(n, k)$. To make this precise we introduce more notation. Let $\varepsilon>0$. Suppose that $H$ and $H^{\prime}$ are $k$-uniform hypergraphs on $n$ vertices. We say that $H$ is $\varepsilon$-close to $H^{\prime}$ if $H$ becomes a copy of $H^{\prime}$ after adding and deleting at most $\varepsilon n^{k}$ edges. More precisely, $H$ is $\varepsilon$-close to $H^{\prime}$ if there is an isomorphic copy $\tilde{H}$ of $H$ such that $V(\tilde{H})=V\left(H^{\prime}\right)$ and $\left|E(\tilde{H}) \triangle E\left(H^{\prime}\right)\right| \leqslant \varepsilon n^{k}$.

Our proof of the non-extremal case uses the absorbing method. Given a $k$-uniform hypergraph $H$, a set $S \subseteq V(H)$ is called an absorbing set for $Q \subseteq V(H)$, if both $H[S]$ and $H[S \cup Q]$ contain perfect matchings. In this case, if the matching covering $S$ is $M$, we also say $M$ absorbs $Q$.

Our main result, Theorem 5, extends [20, Theorem 3.1]. It ensures that if $H$ is as in Theorem 4 then $H$ contains a small absorbing matching or $H$ is close to one of $\mathcal{B}_{n, k}$ and $\overline{\mathcal{B}}_{n, k}$. We postpone its proof to the next subsection.

Theorem 5. Given any $\varepsilon>0$ and integer $k \geqslant 2$, there exist $0<\alpha, \xi<\varepsilon$ and $n_{0} \in \mathbb{N}$ such that the following holds. Suppose that $H$ is a $k$-uniform hypergraph on $n \geqslant n_{0}$ vertices. If

$$
\delta_{1}(H) \geqslant\left(\frac{1}{2}-\alpha\right)\left(\begin{array}{l}
n-1 \\
k-1
\end{array}\right)
$$

then $H$ is $\varepsilon$-close to $\mathcal{B}_{n, k}$ or $\overline{\mathcal{B}}_{n, k}$, or $H$ contains a matching $M$ of size $|M| \leqslant \xi n / k$ that absorbs any set $W \subseteq V(H) \backslash V(M)$ such that $|W| \in k \mathbb{N}$ with $|W| \leqslant \xi^{2} n$.

The next result from [19] ensures a perfect matching when our hypergraph $H$ is close to one of the extremal hypergraphs $\mathcal{B}_{n, k}$ and $\overline{\mathcal{B}}_{n, k}$.

Theorem 6. [19, Theorem 4.1] Given $1 \leqslant \ell \leqslant k-1$, there exist $\varepsilon>0$ and $n_{0} \in \mathbb{N}$ such that the following holds. Suppose that $H$ is a $k$-uniform hypergraph on $n \geqslant n_{0}$ vertices such that $n$ is divisible by $k$. If $\delta_{\ell}(H)>\delta(n, k, \ell)$ and $H$ is $\varepsilon$-close to $\mathcal{B}_{n, k}$ or $\overline{\mathcal{B}}_{n, k}$, then $H$ contains a perfect matching.

The final tool required for the proof of Theorem 4 is a weaker version of Lemma 5.6 in $[11]$.

Lemma 7. [11] Let $k \geqslant 2$ and $1 \leqslant \ell \leqslant k-1$ be integers, and let $\varepsilon>0$. Suppose that for some $b, c \in(0,1)$ and some $n_{0} \in \mathbb{N}$, every $k$-uniform hypergraph $H$ on $n \geqslant n_{0}$ vertices with $\delta_{\ell}(H) \geqslant c n^{k-\ell}$ has a fractional matching of size $(b+\varepsilon) n$. Then there exists an $n_{0}^{\prime} \in \mathbb{N}$ such that any $k$-uniform hypergraph $H$ on $n \geqslant n_{0}^{\prime}$ vertices with $\delta_{\ell}(H) \geqslant(c+\varepsilon) n^{k-\ell}$ contains a matching of size at least bn.

Proof of Theorem 4. Choose $\varepsilon>0$ from Theorem 6. We may additionally assume that $\varepsilon \ll \theta, 1 / k$. Let $0<\alpha, \xi<\varepsilon$ be as in Theorem 5 . Let $n$ be sufficiently large and divisible by $k$. Assume that $H$ is a $k$-uniform hypergraph on $n$ vertices satisfying (2). Since $\delta_{\ell}(H)>\delta(n, k, \ell)=(1 / 2-o(1))\left(\begin{array}{l}n-\ell \\ k-\ell\end{array}\right)$, it follows that $\delta_{1}(H) \geqslant(1 / 2-\alpha)\left(\begin{array}{l}n-1 \\ k-1\end{array}\right)$. By Theorem $5, H$ is $\varepsilon$-close to $\mathcal{B}_{n, k}$ or $\overline{\mathcal{B}}_{n, k}$, or $H$ contains a matching $M$ of size $|M| \leqslant \xi n / k$ 
that absorbs any set $W \subseteq V(H) \backslash V(M)$ satisfying $|W| \in k \mathbb{N}$ with $|W| \leqslant \xi^{2} n$. In the former case, since $\delta_{\ell}(H)>\delta(n, k, \ell)$, Theorem 6 provides a perfect matching in $H$. In the latter case, set $c^{*}:=c_{k, \ell^{\prime}}^{*}, H^{\prime}:=H[V(H) \backslash V(M)]$ and $n_{1}:=\left|V\left(H^{\prime}\right)\right|$. Since $|V(M)| \leqslant \xi n$,

$\delta_{\ell^{\prime}}\left(H^{\prime}\right) \geqslant \delta_{\ell^{\prime}}(H)-|V(M)|\left(\begin{array}{l}n-\ell^{\prime}-1 \\ k-\ell^{\prime}-1\end{array}\right) \geqslant\left(c^{*}+\theta-\xi k\right)\left(\begin{array}{l}n-\ell^{\prime} \\ k-\ell^{\prime}\end{array}\right)>\left(c^{*} /\left(k-\ell^{\prime}\right) !+2 \xi^{2}\right) n_{1}^{k-\ell^{\prime}}$,

where the last inequality follows since $\xi \ll \theta, 1 / k$. Let $c:=c^{*} /\left(k-\ell^{\prime}\right)$ ! $+\xi^{2}$. By the definition of $c^{*}$, for sufficiently large $\tilde{n}$, every $k$-uniform hypergraph $F$ on $\tilde{n}$ vertices with $\delta_{\ell^{\prime}}(F) \geqslant c \tilde{n}^{k-\ell^{\prime}}$ contains a perfect fractional matching. Applying Lemma 7 with $\xi^{2} / k$ and $\left(1-\xi^{2}\right) / k$ playing the roles of $\varepsilon$ and $b$ respectively, we conclude that $H^{\prime}$ contains a matching $M^{\prime}$ of size at least $\left(1-\xi^{2}\right) n_{1} / k$. Let $W$ be the uncovered vertices of $H^{\prime}$. Then $|W| \leqslant \xi^{2} n$. We finally absorb $W$ using the absorbing property of $M$.

\subsection{Proof of Theorem 5}

The proof of Theorem 5 follows from the following three lemmas. Lemma 8 is a special case of [13, Lemma 1.1] and gives a sufficient condition for a hypergraph $H$ to contain a small matching that absorbs any much smaller set of vertices from $H$.

Lemma 8. [13] Let $k \in \mathbb{N}$ and $\gamma^{\prime}>0$. Then there exists an $n_{0} \in \mathbb{N}$ such that the following holds. Suppose that $H$ is a k-uniform hypergraph on $n \geqslant n_{0}$ vertices so that, for any $x, y \in V(H)$, there are at least $\gamma^{\prime} n^{2 k-1}(2 k-1)$-sets $X \subseteq V(H)$ such that both $H[X \cup\{x\}]$ and $H[X \cup\{y\}]$ contain perfect matchings. Then $H$ contains a matching $M$ so that

- $|M| \leqslant\left(\gamma^{\prime} / 2\right)^{k} n /\left(8 k^{2}(k-1)\right)$;

- $M$ absorbs any $W \subseteq V(G) \backslash M$ such that $|W| \in k \mathbb{N}$ and $|W| \leqslant\left(\gamma^{\prime} / 2\right)^{2 k} n /(128 k(k-$ $\left.1)^{2}\right)$.

Lemma 9. Let $k \in \mathbb{N}$ and $0<\gamma^{\prime} \ll \gamma \ll 1 / k$. There exists an $n_{0} \in \mathbb{N}$ such that the following holds. Let $H=(V, E)$ be a k-uniform hypergraph on $n \geqslant n_{0}$ vertices. Suppose that for every $x, y \in V$ at least one of the following conditions holds.

(i) $\left|N_{H}(x) \cap N_{H}(y)\right| \geqslant \gamma n^{k-1}$;

(ii) There exists at least $\gamma n$ vertices $z \in V$ such that $\left|N_{H}(x) \cap N_{H}(z)\right| \geqslant \gamma n^{k-1}$ and $\left|N_{H}(y) \cap N_{H}(z)\right| \geqslant \gamma n^{k-1}$.

There there are at least $\gamma^{\prime} n^{2 k-1}(2 k-1)$-sets $X \subseteq V$ such that both $H[X \cup\{x\}]$ and $H[X \cup\{y\}]$ contain perfect matchings.

Lemma 10. Let $k \in \mathbb{N}$ and $0<\alpha \ll \gamma \ll \varepsilon, 1 / k$. Then there exists an $n_{0} \in \mathbb{N}$ such that the following holds. Let $H=(V, E)$ be a k-uniform hypergraph on $n \geqslant n_{0}$ vertices such that $\delta_{1}(H) \geqslant(1 / 2-\alpha)\left(\begin{array}{l}n-1 \\ k-1\end{array}\right)$. Suppose that there exists $x_{0}, y_{0} \in V$ such that 
(i) $\left|N_{H}\left(x_{0}\right) \cap N_{H}\left(y_{0}\right)\right|<\gamma n^{k-1}$;

(ii) at most $\gamma n$ vertices $z \in V$ satisfy $\left|N_{H}(z) \cap N_{H}\left(x_{0}\right)\right| \geqslant \gamma n^{k-1}$ and $\left|N_{H}(z) \cap N_{H}\left(y_{0}\right)\right| \geqslant$ $\gamma n^{k-1}$.

Then $H$ is $\varepsilon$-close to $\mathcal{B}_{n, k}$ or $\overline{\mathcal{B}}_{n, k}$.

We postpone the proof of Lemmas 9 and 10 and prove Theorem 5 first.

Proof of Theorem 5. Given $\varepsilon>0$ and $k \geqslant 2$, choose constants $\alpha, \gamma^{\prime}, \gamma$ so that $0<\alpha \ll$ $\gamma^{\prime} \ll \gamma \ll \varepsilon, 1 / k$. Set $\xi:=\left(\gamma^{\prime} / 2\right)^{k} / \sqrt{128 k(k-1)^{2}}$. Let $n$ be sufficiently large and $H$ be a $k$-uniform hypergraph as in the statement of the theorem.

By Lemmas 9 and 10, $H$ is $\varepsilon$-close to $\mathcal{B}_{n, k}$ or $\overline{\mathcal{B}}_{n, k}$ or for every $x, y \in V(H)$ there are at least $\gamma^{\prime} n^{2 k-1}(2 k-1)$-sets $X \subseteq V(H)$ such that both $H[X \cup\{x\}]$ and $H[X \cup\{y\}]$ contain perfect matchings. In the former case we are done. In the latter case, Lemma 8 implies that $H$ contains a matching $M$ so that

- $|M| \leqslant\left(\gamma^{\prime} / 2\right)^{k} n /\left(8 k^{2}(k-1)\right) \leqslant \xi n / k$;

- $M$ absorbs any $W \subseteq V(H) \backslash M$ such that $|W| \in k \mathbb{N}$ and $|W| \leqslant\left(\gamma^{\prime} / 2\right)^{2 k} n /(128 k(k-$ $\left.1)^{2}\right)=\xi^{2} n$,

as required.

Proof of Lemma 9. Note that (i) and (ii) imply that $\delta_{1}(H) \geqslant \gamma n^{k-1}$ and so $e(H) \geqslant$ $\gamma n^{k} / k$. Consider any $x, y \in V$. First assume that (i) holds. Fix any $X^{\prime} \subseteq V$ where $\left|X^{\prime}\right|=k-1$ and $X^{\prime} \cup\{x\}, X^{\prime} \cup\{y\} \in E$. By (i) there are at least $\gamma n^{k-1}$ choices for $X^{\prime}$. Next choose some $X^{\prime \prime} \subseteq V \backslash\left(X^{\prime} \cup\{x, y\}\right)$ such that $\left|X^{\prime \prime}\right|=k$ and $X^{\prime \prime} \in E$. There are at least $\gamma n^{k} / k-(k+1)\left(\begin{array}{c}n \\ k-1\end{array}\right) \geqslant \gamma n^{k} /(2 k)$ choices for $X^{\prime \prime}$. Set $X:=X^{\prime} \cup X^{\prime \prime}$. Note that both $H[X \cup\{x\}]$ and $H[X \cup\{y\}]$ contain perfect matchings. Further, since there are at least $\gamma n^{k-1}$ choices for $X^{\prime}$, at least $\gamma n^{k} /(2 k)$ choices for $X^{\prime \prime}$ and each $(2 k-1)$-set may be counted at most $\left(\begin{array}{c}2 k-1 \\ k-1\end{array}\right)$ times, there are at least

$$
\gamma n^{k-1} \times \frac{\gamma n^{k}}{2 k} \times \frac{1}{\left(\begin{array}{c}
2 k-1 \\
k-1
\end{array}\right)}>\gamma^{\prime} n^{2 k-1}
$$

choices for $X$ (as $\left.\gamma^{\prime} \ll \gamma \ll 1 / k\right)$, as desired.

Now suppose that (ii) holds. Fix any $z \in V$ such that $\left|N_{H}(x) \cap N_{H}(z)\right| \geqslant \gamma n^{k-1}$ and $\left|N_{H}(y) \cap N_{H}(z)\right| \geqslant \gamma n^{k-1}$. There are at least $\gamma n$ choices for $z$. Next fix some $X^{\prime} \in N_{H}(x) \cap N_{H}(z)$ that is disjoint from $y$. There are at least $\gamma n^{k-1}-\left(\begin{array}{c}n \\ k-2\end{array}\right) \geqslant \gamma n^{k-1} / 2$ choices for $X^{\prime}$. Finally, fix some $X^{\prime \prime} \in N_{H}(y) \cap N_{H}(z)$ so that $X^{\prime \prime}$ is disjoint from $X^{\prime} \cup\{x\}$. There are at least $\gamma n^{k-1}-k\left(\begin{array}{c}n \\ k-2\end{array}\right) \geqslant \gamma n^{k-1} / 2$ choices for $X^{\prime \prime}$. Set $X:=X^{\prime} \cup X^{\prime \prime} \cup\{z\}$. So $|X|=2 k-1$ and both $H[X \cup\{x\}]$ and $H[X \cup\{y\}]$ contain perfect matchings. Further, there are at least

$$
\gamma n \times \frac{\gamma n^{k-1}}{2} \times \frac{\gamma n^{k-1}}{2} \times \frac{1}{(2 k-1)\left(\begin{array}{c}
2 k-2 \\
k-1
\end{array}\right)}>\gamma^{\prime} n^{2 k-1}
$$

choices for $X\left(\right.$ as $\left.\gamma^{\prime} \ll \gamma \ll 1 / k\right)$, as desired. 
The rest of this section is devoted to the proof of Lemma 10. We draw on ideas used in the proof of Lemma 5.4 in [20]. We need two results from [20]. The first one implies that if any two vertices in a hypergraph have roughly the same neighborhood, then the hypergraph is near complete or empty.

Lemma 11. [20, Lemma 2.2] Given any $k \in \mathbb{N}$ and $\rho>0$ there exists an $n_{0} \in \mathbb{N}$ such that the following holds. Let $F=(V, E)$ be a $k$-uniform hypergraph on $n \geqslant n_{0}$ vertices with edge density $|E| /\left(\begin{array}{l}n \\ k\end{array}\right) \in[\rho, 1-\rho]$. Then there exist two vertices $v, v^{\prime} \in V$ such that $\left|N_{F}(v) \triangle N_{F}\left(v^{\prime}\right)\right| \geqslant \rho(1-\rho) n^{k-1} /(k+1)$ !.

Proposition 12. [20, Proposition 2.3] For $r \in \mathbb{N}, 0 \leqslant c \leqslant 1$ and $n \rightarrow \infty$,

$$
\begin{aligned}
\sum_{0 \leqslant i \leqslant r, i \text { even }}\left(\begin{array}{c}
c n \\
r-i
\end{array}\right)\left(\begin{array}{c}
(1-c) n \\
i
\end{array}\right) & =\frac{n^{r}}{2 r !}\left(1+(2 c-1)^{r}\right)-O\left(n^{r-1}\right), \\
\sum_{0 \leqslant i \leqslant r, i \text { odd }}\left(\begin{array}{c}
c n \\
r-i
\end{array}\right)\left(\begin{array}{c}
(1-c) n \\
i
\end{array}\right) & =\frac{n^{r}}{2 r !}\left(1-(2 c-1)^{r}\right)-O\left(n^{r-1}\right) .
\end{aligned}
$$

Proof of Lemma 10. Define

$X:=\left\{v \in V:\left|N_{H}\left(y_{0}\right) \cap N_{H}(v)\right|<\gamma n^{k-1}\right\} \quad$ and $\quad Y:=\left\{v \in V:\left|N_{H}\left(x_{0}\right) \cap N_{H}(v)\right|<\gamma n^{k-1}\right\}$.

Then by Lemma 10 (i), $x_{0} \in X$ and $y_{0} \in Y$. Let $V_{0}:=V \backslash(X \cup Y)$. We have $\left|V_{0}\right| \leqslant \gamma n$ by Lemma 10 (ii). Roughly speaking, our goal is to show that $|X| \approx|Y| \approx n / 2$ and $H \approx \mathcal{B}_{n, k}(X, Y)$ or $H \approx \overline{\mathcal{B}}_{n, k}(X, Y)$.

We first provide several properties of $X$ and $Y$, for example, $X \cap Y=\emptyset$, and $N_{H}(v) \approx$ $N_{H}\left(v^{\prime}\right)$ whenever $v, v^{\prime} \in X$ or $v, v^{\prime} \in Y$.

Claim 13. The following conditions hold.

(i) For all $v \in X \cup Y$, we have $d_{H}(v) \leqslant(1 / 2+\alpha)\left(\begin{array}{l}n-1 \\ k-1\end{array}\right)+\gamma n^{k-1}$.

(ii) $X \cap Y=\emptyset$.

(iii) For any two vertices $x, x^{\prime} \in X$, we have $\left|N_{H}(x) \triangle N_{H}\left(x^{\prime}\right)\right|<5 \gamma n^{k-1}$. The same holds for all $y, y^{\prime} \in Y$.

(iv) For any $x \in X$ and $y \in Y$, we have $\left|N_{H}(x) \cap N_{H}(y)\right| \leqslant 4 \gamma n^{k-1}$ and $\mid \bar{N}_{H}(x) \cap$ $\bar{N}_{H}(y) \mid \leqslant 4 \gamma n^{k-1}$, where $\bar{N}_{H}(x):=\left(\begin{array}{c}V \backslash\{x\} \\ k-1\end{array}\right) \backslash N_{H}(x)$ consists of non-neighbors of $x$.

Proof. To see (i), suppose $x \in X$ and $y \in Y$. Since $d_{H}\left(x_{0}\right), d_{H}\left(y_{0}\right) \geqslant(1 / 2-\alpha)\left(\begin{array}{l}n-1 \\ k-1\end{array}\right)$, by the definition of $X$ and $Y$,

$$
\left|N_{H}\left(y_{0}\right) \backslash N_{H}(x)\right|,\left|N_{H}\left(x_{0}\right) \backslash N_{H}(y)\right| \geqslant\left(\frac{1}{2}-\alpha\right)\left(\begin{array}{l}
n-1 \\
k-1
\end{array}\right)-\gamma n^{k-1} .
$$

Consequently, we have $d_{H}(x), d_{H}(y) \leqslant(1 / 2+\alpha)\left(\begin{array}{l}n-1 \\ k-1\end{array}\right)+\gamma n^{k-1}$. 
To see (ii), suppose that there exists $v \in X \cap Y$. Then by (3),

$$
\begin{aligned}
\left|\left(N_{H}\left(x_{0}\right) \cup N_{H}\left(y_{0}\right)\right) \backslash N_{H}(v)\right| & \geqslant\left|N_{H}\left(x_{0}\right) \backslash N_{H}(v)\right|+\left|N_{H}\left(y_{0}\right) \backslash N_{H}(v)\right|-\left|N_{H}\left(x_{0}\right) \cap N_{H}\left(y_{0}\right)\right| \\
& \geqslant 2\left(\frac{1}{2}-\alpha\right)\left(\begin{array}{l}
n-1 \\
k-1
\end{array}\right)-3 \gamma n^{k-1} \geqslant\left(\begin{array}{l}
n-1 \\
k-1
\end{array}\right)-4 \gamma n^{k-1},
\end{aligned}
$$

which implies that $\left|N_{H}(v)\right| \leqslant 4 \gamma n^{k-1}$, contradicting the minimum degree condition of $H$.

To see (iii), consider $x \in X$. By the definition of $X$ and the minimum degree condition of $H$, we have $\left|N_{H}(x) \cup N_{H}\left(y_{0}\right)\right| \geqslant 2\left(\frac{1}{2}-\alpha\right)\left(\begin{array}{l}n-1 \\ k-1\end{array}\right)-\gamma n^{k-1}$. Let $x^{\prime} \in X \backslash\{x\}$. Then

$$
\begin{aligned}
\left|N_{H}\left(x^{\prime}\right) \backslash N_{H}(x)\right| & \left.=\mid\left(N_{H}\left(x^{\prime}\right) \backslash N_{H}(x)\right) \backslash N_{H}\left(y_{0}\right)\right)|+|\left(N_{H}\left(x^{\prime}\right) \backslash N_{H}(x)\right) \cap N_{H}\left(y_{0}\right) \mid \\
& \leqslant\left|\left(\begin{array}{c}
V \backslash\left\{x^{\prime}\right\} \\
k-1
\end{array}\right) \backslash\left(N_{H}(x) \cup N_{H}\left(y_{0}\right)\right)\right|+\left|N_{H}\left(x^{\prime}\right) \cap N_{H}\left(y_{0}\right)\right| \\
& \leqslant\left(\begin{array}{c}
n-1 \\
k-1
\end{array}\right)-2\left(\frac{1}{2}-\alpha\right)\left(\begin{array}{c}
n-1 \\
k-1
\end{array}\right)+\gamma n^{k-1}+\gamma n^{k-1} \\
& =2 \alpha\left(\begin{array}{c}
n-1 \\
k-1
\end{array}\right)+2 \gamma n^{k-1} .
\end{aligned}
$$

The same bound holds for $\left|N_{H}(x) \backslash N_{H}\left(x^{\prime}\right)\right|$. Hence

$$
\begin{aligned}
\left|N_{H}(x) \triangle N_{H}\left(x^{\prime}\right)\right| & =\left|N_{H}\left(x^{\prime}\right) \backslash N_{H}(x)\right|+\left|N_{H}(x) \backslash N_{H}\left(x^{\prime}\right)\right| \\
& \leqslant 2\left(2 \alpha\left(\begin{array}{l}
n-1 \\
k-1
\end{array}\right)+2 \gamma n^{k-1}\right)<5 \gamma n^{k-1} .
\end{aligned}
$$

Analogously we can derive that $\left|N_{H}(y) \triangle N_{H}\left(y^{\prime}\right)\right|<5 \gamma n^{k-1}$ for any $y, y^{\prime} \in Y$.

To see (iv), consider $x \in X$ and $y \in Y$. By (4), we have $\left|N_{H}(x) \backslash N_{H}\left(x_{0}\right)\right| \leqslant$ $2 \alpha\left(\begin{array}{l}n-1 \\ k-1\end{array}\right)+2 \gamma n^{k-1}$. By the definition of $Y$, we have $\left|N_{H}(y) \cap N_{H}\left(x_{0}\right)\right|<\gamma n^{k-1}$. Thus

$$
\begin{aligned}
\left|N_{H}(x) \cap N_{H}(y)\right| & \leqslant\left|N_{H}(y) \cap N_{H}\left(x_{0}\right)\right|+\left|N_{H}(x) \backslash N_{H}\left(x_{0}\right)\right| \\
& \leqslant 2 \alpha\left(\begin{array}{l}
n-1 \\
k-1
\end{array}\right)+3 \gamma n^{k-1} \leqslant 4 \gamma n^{k-1},
\end{aligned}
$$

which proves the first assertion of (iv). By the minimum degree condition and (5), we have

$$
\left|N_{H}(x) \cup N_{H}(y)\right| \geqslant 2\left(\frac{1}{2}-\alpha\right)\left(\begin{array}{l}
n-1 \\
k-1
\end{array}\right)-3 \gamma n^{k-1}-2 \alpha\left(\begin{array}{l}
n-1 \\
k-1
\end{array}\right) .
$$

It follows that

$$
\begin{aligned}
\left|\bar{N}_{H}(x) \cap \bar{N}_{H}(y)\right| & \leqslant\left(\begin{array}{c}
|V \backslash\{x, y\}| \\
k-1
\end{array}\right)-\left|N_{H}(x) \cup N_{H}(y)\right| \\
& \leqslant\left(\begin{array}{c}
n-2 \\
k-1
\end{array}\right)-(1-2 \alpha)\left(\begin{array}{c}
n-1 \\
k-1
\end{array}\right)+3 \gamma n^{k-1}+2 \alpha\left(\begin{array}{c}
n-1 \\
k-1
\end{array}\right) \leqslant 4 \gamma n^{k-1},
\end{aligned}
$$

which proves the second assertion of (iv). 
Since $\left|V_{0}\right| \leqslant \gamma n$ and $X \cap Y=\emptyset$ we have $|X| \geqslant(1-\gamma) n / 2$ or $|Y| \geqslant(1-\gamma) n / 2$. Without loss of generality we may assume that $|X| \geqslant(1-\gamma) n / 2 \geqslant n / 3$. Let $0<\gamma_{0}<1 / 2$ such that $\frac{\gamma_{0}\left(1-\gamma_{0}\right)}{(k+1) !}=5 \gamma \cdot 3^{k-1}$. We apply Lemma 11 to $F=H[X]$ with $\rho=\gamma_{0}$. Since

$$
\left|N_{F}(v) \triangle N_{F}\left(v^{\prime}\right)\right| \leqslant\left|N_{H}(v) \triangle N_{H}\left(v^{\prime}\right)\right|<5 \gamma n^{k-1} \leqslant \frac{\gamma_{0}\left(1-\gamma_{0}\right)}{(k+1) !}|X|^{k-1}
$$

for any $v, v^{\prime} \in X$ (Claim 13 (iii)), there are two possible cases:

Case $1 e(H[X]) \leqslant \gamma_{0}\left(\begin{array}{c}|X| \\ k\end{array}\right)$,

Case $2 e(H[X]) \geqslant\left(1-\gamma_{0}\right)\left(\begin{array}{c}|X| \\ k\end{array}\right)$.

In the rest of the proof we assume that one of the two cases holds. Once we have obtained more information we will prove that $|X|$ and $|Y|$ are close to $n / 2$. At present we require the following weaker lower bounds on $|X|$ and $|Y|$.

Claim 14. $|X|,|Y| \geqslant\left(1-\left(\frac{1}{2}+2 \gamma_{0}\right)^{\frac{1}{k-1}}-\gamma\right) n$.

Proof. The bound on $|X|$ follows since $|X| \geqslant(1-\gamma) n / 2$. Since $|X|+|Y|+\left|V_{0}\right|=n$ and $\left|V_{0}\right| \leqslant \gamma n$, to prove the bound on $|Y|$, it suffices to show that $|X| \leqslant\left(\frac{1}{2}+2 \gamma_{0}\right)^{\frac{1}{k-1}} n$. In Case 1 , there exists a vertex $x \in X$ such that $d_{H[X]}(x) \leqslant \gamma_{0}\left(\begin{array}{c}|X|-1 \\ k-1\end{array}\right)$ and consequently, $\left|\bar{N}_{H[X]}(x)\right| \geqslant\left(1-\gamma_{0}\right)\left(\begin{array}{c}|X|-1 \\ k-1\end{array}\right)$. Together with the minimum degree condition, this gives

$$
\left(\frac{1}{2}-\alpha\right)\left(\begin{array}{l}
n-1 \\
k-1
\end{array}\right) \leqslant d_{H}(x) \leqslant\left(\begin{array}{l}
n-1 \\
k-1
\end{array}\right)-\left(1-\gamma_{0}\right)\left(\begin{array}{c}
|X|-1 \\
k-1
\end{array}\right) .
$$

In Case 2, there exists a vertex $x \in X$ such that $d_{H[X]}(x) \geqslant\left(1-\gamma_{0}\right)\left(\begin{array}{c}|X|-1 \\ k-1\end{array}\right)$. By Claim 13 (i),

$$
\left(1-\gamma_{0}\right)\left(\begin{array}{c}
|X|-1 \\
k-1
\end{array}\right) \leqslant d_{H[X]}(x) \leqslant\left(\frac{1}{2}+\alpha\right)\left(\begin{array}{l}
n-1 \\
k-1
\end{array}\right)+\gamma n^{k-1}
$$

In either case we have $\left(1-\gamma_{0}\right)\left(\begin{array}{c}|X|-1 \\ k-1\end{array}\right) \leqslant\left(\frac{1}{2}+\alpha\right)\left(\begin{array}{c}n-1 \\ k-1\end{array}\right)+\gamma n^{k-1}$, which implies that $\left(\begin{array}{c}|X|-1 \\ k-1\end{array}\right) \leqslant$ $\left(1+2 \gamma_{0}\right)\left((1 / 2+\alpha)\left(\begin{array}{l}n-1 \\ k-1\end{array}\right)+\gamma n^{k-1}\right)$. Letting $|X|=c n$, it follows that

$$
c^{k-1}\left(\begin{array}{l}
n-1 \\
k-1
\end{array}\right)-O\left(n^{k-2}\right) \leqslant\left(\frac{1}{2}+\gamma_{0}+2 \alpha\right)\left(\begin{array}{l}
n-1 \\
k-1
\end{array}\right)+2 \gamma n^{k-1}
$$

Since $\gamma n^{k-1} \leqslant \frac{\gamma_{0}}{5}\left(\begin{array}{l}n-1 \\ k-1\end{array}\right)$, we conclude that $c \leqslant\left(\frac{1}{2}+2 \gamma_{0}\right)^{\frac{1}{k-1}}$.

Given two disjoint subsets $A, B \subset V$ and two integers $i, j \geqslant 0$, we call an $(i+j)$-set $S \subseteq V$ an $A^{i} B^{j}$-set if $|S \cap A|=i$ and $|S \cap B|=j$, and let $A^{i} B^{j}$ denote the family of all $A^{i} B^{j}$-sets. Let $c_{0}:=1-\left(\frac{1}{2}+2 \gamma_{0}\right)^{\frac{1}{k-1}}-\gamma$, and $\gamma_{i}:=\gamma_{i-1}+5 \gamma k ! / c_{0}^{k-1}$ for $i=1, \ldots, k$.

Claim 15. (i) In Case 1 , for $1 \leqslant i \leqslant k$, for any $y \in Y$, at least $\left(1-\gamma_{i}\right)\left(\begin{array}{c}|X| \\ k-i\end{array}\right)\left(\begin{array}{c}|Y| \\ i-1\end{array}\right)$ $X^{k-i} Y^{i-1}$-sets are neighbors (respectively, non-neighbors) of $y$ if $i$ is odd (respectively, even). 
(ii) In Case 2, for $1 \leqslant i \leqslant k$, for any $y \in Y$, at least $\left(1-\gamma_{i}\right)\left(\begin{array}{c}|X| \\ k-i\end{array}\right)\left(\begin{array}{c}|Y| \\ i-1\end{array}\right) X^{k-i} Y^{i-1}$-sets are neighbors (respectively, non-neighbors) of $y$ if $i$ is even (respectively, odd).

Proof. We prove both cases by induction on $i$. In Case 1 there exists a vertex $x_{1} \in X$ such that $d_{H[X]}\left(x_{1}\right) \leqslant \gamma_{0}\left(\begin{array}{c}|X|-1 \\ k-1\end{array}\right)$ and consequently $\left|\bar{N}_{H}\left(x_{1}\right) \cap\left(\begin{array}{c}X \\ k-1\end{array}\right)\right| \geqslant\left(1-\gamma_{0}\right)\left(\begin{array}{c}|X|-1 \\ k-1\end{array}\right)$. Fix a vertex $y \in Y$. By Claim 13 (iv), $\left|\bar{N}_{H}\left(x_{1}\right) \cap \bar{N}_{H}(y)\right| \leqslant 4 \gamma n^{k-1}$. Thus at least $\left(1-\gamma_{0}\right)\left(\begin{array}{c}|X|-1 \\ k-1\end{array}\right)-4 \gamma n^{k-1} X^{k-1}$-sets are neighbors of $y$. By Claim $14,|X|,|Y| \geqslant c_{0} n$. Then for any $0 \leqslant i \leqslant k-1$,

$$
\left(\begin{array}{c}
|X| \\
k-i-1
\end{array}\right)\left(\begin{array}{c}
|Y| \\
i
\end{array}\right) \geqslant \frac{\left(c_{0} n\right)^{k-1}}{(k-1) !}-O\left(n^{k-2}\right) \geqslant \frac{c_{0}^{k-1} n^{k-1}}{k !} .
$$

Together with the definition of $\gamma_{1}$, we conclude that at least

$$
\left(1-\gamma_{0}\right)\left(\begin{array}{c}
|X|-1 \\
k-1
\end{array}\right)-4 \gamma n^{k-1} \geqslant\left(1-\gamma_{0}\right)\left(\begin{array}{c}
|X| \\
k-1
\end{array}\right)-O\left(n^{k-2}\right)-\frac{4 \gamma k !}{c_{0}^{k-1}}\left(\begin{array}{c}
|X| \\
k-1
\end{array}\right) \geqslant\left(1-\gamma_{1}\right)\left(\begin{array}{c}
|X| \\
k-1
\end{array}\right)
$$

$X^{k-1}$-sets are neighbors of $y$. This confirms (i) for $i=1$. In Case 2, by averaging, there exists a vertex $x_{1} \in X$ such that $d_{H[X]}\left(x_{1}\right) \geqslant\left(1-\gamma_{0}\right)\left(\begin{array}{c}|X|-1 \\ k-1\end{array}\right)$. Fix a vertex $y \in Y$. By Claim 13 (iv), $\left|N_{H}\left(x_{1}\right) \cap N_{H}(y)\right| \leqslant 4 \gamma n^{k-1}$. Thus at least $\left(1-\gamma_{0}\right)\left(\begin{array}{c}|X|-1 \\ k-1\end{array}\right)-4 \gamma n^{k-1} \geqslant$ $\left(1-\gamma_{1}\right)\left(\begin{array}{c}|X| \\ k-1\end{array}\right) X^{k-1}$-sets are non-neighbors of $y$. This confirms (ii) for $i=1$.

For the induction step, we first assume that for some $1 \leqslant i \leqslant k$, every $y \in Y$ has at least $\left(1-\gamma_{i}\right)\left(\begin{array}{c}|X| \\ k-i\end{array}\right)\left(\begin{array}{l}|Y| \\ i-1\end{array}\right) X^{k-i} Y^{i-1}$-sets in its neighborhood. Consequently at least $\left(1-\gamma_{i}\right)\left(\begin{array}{c}|X| \\ k-i\end{array}\right)\left(\begin{array}{c}|Y| \\ i\end{array}\right) X^{k-i} Y^{i}$-sets are edges of $H$. By averaging, there exists $x_{i} \in X$ whose neighborhood contains at least $\left(1-\gamma_{i}\right)\left(\begin{array}{c}|X|-1 \\ k-i-1\end{array}\right)\left(\begin{array}{c}|Y| \\ i\end{array}\right) X^{k-i-1} Y^{i}$-sets. Fix $y \in Y$. By Claim 13 (iv),

$$
\left|\left(N_{H}\left(x_{i}\right) \cap X^{k-i-1} Y^{i}\right) \backslash N_{H}(y)\right| \geqslant\left(1-\gamma_{i}\right)\left(\begin{array}{c}
|X|-1 \\
k-i-1
\end{array}\right)\left(\begin{array}{c}
|Y| \\
i
\end{array}\right)-4 \gamma n^{k-1}
$$

Since $\left|\bar{N}_{H}(y) \cap X^{k-i-1} Y^{i}\right| \geqslant\left|\left(N_{H}\left(x_{i}\right) \cap X^{k-i-1} Y^{i}\right) \backslash N_{H}(y)\right|-O\left(n^{k-2}\right)$, we conclude that at least

$$
\left(1-\gamma_{i}\right)\left(\begin{array}{c}
|X|-1 \\
k-i-1
\end{array}\right)\left(\begin{array}{c}
|Y| \\
i
\end{array}\right)-4 \gamma n^{k-1}-O\left(n^{k-2}\right) \geqslant\left(1-\gamma_{i+1}\right)\left(\begin{array}{c}
|X| \\
k-i-1
\end{array}\right)\left(\begin{array}{c}
|Y| \\
i
\end{array}\right)
$$

$X^{k-i-1} Y^{i}$-sets are non-neighbors of $y$, where we use (6) and the definition of $\gamma_{i+1}$. Analogously we can show that if for some $1 \leqslant i \leqslant k$, every $y \in Y$ has at least $\left(1-\gamma_{i}\right)\left(\begin{array}{c}|X| \\ k-i\end{array}\right)\left(\begin{array}{l}|Y| \\ i-1\end{array}\right)$ $X^{k-i} Y^{i-1}$-sets as non-neighbors, then at least $\left(1-\gamma_{i+1}\right)\left(\begin{array}{c}|X| \\ k-i-1\end{array}\right)\left(\begin{array}{c}|Y| \\ i\end{array}\right) X^{k-i-1} Y^{i}$-sets are neighbors of $y$. This completes our induction proof.

Claim 16. $|X|,|Y| \geqslant(1-\eta) n / 2$, where $\eta:=\left(2 \gamma_{k}\right)^{1 /(k-1)}+\gamma$. 
Proof. Suppose that Claim 15 (i) holds (the proof when Claim 15 (ii) holds is analogous).

Let $\tilde{n}:=|X \cup Y|$. Note that $\tilde{n} \geqslant(1-\gamma) n$ because $\left|V_{0}\right| \leqslant \gamma n$. Let $c:=|X| / \tilde{n}$. It suffices to show that $\left(1-\left(2 \gamma_{k}\right)^{1 /(k-1)}\right) / 2 \leqslant c \leqslant\left(1+\left(2 \gamma_{k}\right)^{1 /(k-1)}\right) / 2$ because this implies that

$$
|X|=c \tilde{n} \geqslant \frac{1}{2}\left(1-\left(2 \gamma_{k}\right)^{1 /(k-1)}\right)(1-\gamma) n>(1-\eta) \frac{n}{2}
$$

and $|Y|=(1-c) \tilde{n} \geqslant \frac{1}{2}\left(1-\left(2 \gamma_{k}\right)^{1 /(k-1)}\right)(1-\gamma) n>(1-\eta) n / 2$.

For any $y \in Y$, by Claim 15 (i),

$$
d_{H}(y) \geqslant \sum_{1 \leqslant i \leqslant k, i \text { odd }}\left(1-\gamma_{i}\right)\left(\begin{array}{c}
|X| \\
k-i
\end{array}\right)\left(\begin{array}{c}
|Y| \\
i-1
\end{array}\right) \geqslant\left(1-\gamma_{k}\right) \sum_{0 \leqslant j \leqslant k-1, j \text { even }}\left(\begin{array}{c}
|X| \\
k-1-j
\end{array}\right)\left(\begin{array}{c}
|Y| \\
j
\end{array}\right) .
$$

Hence, by Proposition $12, d_{H}(y) \geqslant\left(1-\gamma_{k}\right) \frac{\tilde{n}^{k-1}}{2(k-1) !}\left(1+(2 c-1)^{k-1}\right)-O\left(n^{k-2}\right)$. If $(2 c-1)^{k-1} \geqslant$ $2 \gamma_{k}$, then

$$
d_{H}(y) \geqslant\left(1-\gamma_{k}\right) \frac{(1-\gamma)^{k-1} n^{k-1}}{2(k-1) !}\left(1+2 \gamma_{k}\right)-O\left(n^{k-2}\right) \geqslant\left(1+\frac{\gamma_{k}}{2}\right) \frac{n^{k-1}}{2(k-1) !},
$$

as $5 \gamma(k-1) !<\gamma_{k} \ll 1$. This contradicts Claim 13 (i). Thus $(2 c-1)^{k-1}<2 \gamma_{k}$. If $c \geqslant 1 / 2$, then $c<\left(1+\left(2 \gamma_{k}\right)^{1 /(k-1)}\right) / 2$; if $c<1 / 2$ and $k-1$ is even, then $(1-2 c)^{k-1}=(2 c-1)^{k-1}<$ $2 \gamma_{k}$ and thus $c>\left(1-\left(2 \gamma_{k}\right)^{1 /(k-1)}\right) / 2$. In either case we are done. Otherwise assume that $c<1 / 2$ and $k-1$ is odd. By Claim 15 (i),

$$
\bar{d}_{H}(y) \geqslant \sum_{1 \leqslant i \leqslant k, i \text { even }}\left(1-\gamma_{i}\right)\left(\begin{array}{c}
|X| \\
k-i
\end{array}\right)\left(\begin{array}{c}
|Y| \\
i-1
\end{array}\right) \geqslant\left(1-\gamma_{k}\right) \sum_{0 \leqslant j \leqslant k-1, j \text { odd }}\left(\begin{array}{c}
|X| \\
k-1-j
\end{array}\right)\left(\begin{array}{c}
|Y| \\
j
\end{array}\right),
$$

where $\bar{d}_{H}(y):=\left|\bar{N}_{H}(y)\right|$. By Proposition 12 , we have $\bar{d}_{H}(y) \geqslant\left(1-\gamma_{k}\right) \frac{\tilde{n}^{k-1}}{2(k-1) !}(1-(2 c-$ $\left.1)^{k-1}\right)-O\left(n^{k-2}\right)$. If $1-(2 c-1)^{k-1}=1+(1-2 c)^{k-1} \geqslant 1+2 \gamma_{k}$, then we obtain a contradiction as before because $\bar{d}_{H}(y) \leqslant\left(\frac{1}{2}+\alpha\right)\left(\begin{array}{l}n-1 \\ k-1\end{array}\right)$. Hence, $(1-2 c)^{k-1}<2 \gamma_{k}$ and consequently $c>\left(1-\left(2 \gamma_{k}\right)^{1 /(k-1)}\right) / 2$, as desired.

By Claim 16, there exists a partition $X^{\prime}, Y^{\prime}$ of $V$ such that $\left|X^{\prime}\right|=\lceil n / 2\rceil,\left|Y^{\prime}\right|=$ $\lfloor n / 2\rfloor$ and $\left|X \cap X^{\prime}\right|,\left|Y \cap Y^{\prime}\right| \geqslant(1-\eta) n / 2$. We claim that $H$ is $\varepsilon$-close to $\mathcal{B}_{n, k}\left(Y^{\prime}, X^{\prime}\right)$ in Case 1 and $\varepsilon$-close to $\overline{\mathcal{B}}_{n, k}\left(Y^{\prime}, X^{\prime}\right)$ in Case 2. Indeed, set $\mathcal{B}:=\mathcal{B}_{\tilde{n}, k}(Y, X)$, where $\tilde{n}:=|X \cup Y|$, and $H^{\prime}:=H[X \cup Y]$. By definition, $E(\mathcal{B})$ consists of all $X^{k-i} Y^{i}$-sets for all odd $0 \leqslant i \leqslant k$. If Claim 15 (i) holds, then $\left|E(\mathcal{B}) \cap E\left(H^{\prime}\right)\right| \geqslant\left(1-\gamma_{k}\right)|E(\mathcal{B})|$ and $\left|E(\overline{\mathcal{B}}) \cap E\left(\overline{H^{\prime}}\right)\right| \geqslant\left(1-\gamma_{k}\right)|E(\overline{\mathcal{B}})|$. Thus

$$
\left|E(\mathcal{B}) \triangle E\left(H^{\prime}\right)\right|=\left|E(\mathcal{B}) \backslash E\left(H^{\prime}\right)\right|+\left|E(\overline{\mathcal{B}}) \backslash E\left(\overline{H^{\prime}}\right)\right| \leqslant \gamma_{k}|E(\mathcal{B})|+\gamma_{k}|E(\overline{\mathcal{B}})| \leqslant \gamma_{k}\left(\begin{array}{l}
n \\
k
\end{array}\right) .
$$

Let $V^{\prime}:=\left(X \cap X^{\prime}\right) \cup\left(Y \cap Y^{\prime}\right)$. Then $\left|V^{\prime}\right| \geqslant(1-\eta) n$ and

$$
\left|\left(E\left(\mathcal{B}_{n, k}\left(Y^{\prime}, X^{\prime}\right)\right) \triangle E(H)\right) \backslash\left(\begin{array}{c}
V^{\prime} \\
k
\end{array}\right)\right| \leqslant \eta n\left(\begin{array}{c}
n-1 \\
k-1
\end{array}\right) .
$$


Since $\gamma \ll \varepsilon, 1 / k$, we have that $\gamma_{k}, \eta \ll \varepsilon$. Therefore,

$$
\left|E\left(\mathcal{B}_{n, k}\left(Y^{\prime}, X^{\prime}\right)\right) \triangle E(H)\right| \leqslant \eta n\left(\begin{array}{l}
n-1 \\
k-1
\end{array}\right)+\left|E(\mathcal{B}) \triangle E\left(H^{\prime}\right)\right| \leqslant \eta n\left(\begin{array}{l}
n-1 \\
k-1
\end{array}\right)+\gamma_{k}\left(\begin{array}{l}
n \\
k
\end{array}\right) \leqslant \varepsilon\left(\begin{array}{l}
n \\
k
\end{array}\right) .
$$

which implies that $H$ is $\varepsilon$-close to $\mathcal{B}_{n, k}\left(Y^{\prime}, X^{\prime}\right)$. Analogously we can show that $H$ is $\varepsilon$-close to $\overline{\mathcal{B}}_{n, k}\left(Y^{\prime}, X^{\prime}\right)$ in Case 2. This completes the proof of Lemma 10.

\section{An application of Lemma 9}

The following simple application of Lemma 9 implies that the minimum $\ell$-degree condition that forces a perfect fractional matching also forces a perfect matching in a $k$-uniform hypergraph $H$, if we additionally assume that $H$ has a small number of vertices of large degree.

Theorem 17. Given any $0<\varepsilon \leqslant \delta^{\prime}$ and $k, \ell \in \mathbb{N}$ where $\ell<k$, there is an $n_{0} \in \mathbb{N}$ such that the following holds. Let $H$ be a $k$-uniform hypergraph on $n \geqslant n_{0}$ vertices where $k$ divides $n$. Suppose that $\delta_{1}(H) \geqslant \delta^{\prime}\left(\begin{array}{l}n-1 \\ k-1\end{array}\right)$ and $\delta_{\ell}(H) \geqslant\left(c_{k, \ell}^{*}+\varepsilon\right)\left(\begin{array}{l}n-\ell \\ k-\ell\end{array}\right)$. If there are at least $\varepsilon$ vertices $x \in V(H)$ so that $d_{H}(x) \geqslant\left(1-\delta^{\prime}+\varepsilon\right)\left(\begin{array}{l}n-1 \\ k-1\end{array}\right)$ then $H$ contains a perfect matching.

Sketch proof. It is easy to see that $H$ satisfies Lemma 9 (ii) (where we choose $0<\gamma \ll \varepsilon$ ) and so by Lemma 8, $H$ contains a small absorbing matching $M$. Let $H^{\prime}:=H \backslash V(M)$. Then $\delta_{\ell}\left(H^{\prime}\right) \geqslant\left(c_{k, \ell}^{*}+\varepsilon / 2\right)\left(\begin{array}{l}n-\ell \\ k-\ell\end{array}\right)$ and so by Lemma $7, H^{\prime}$ contains a matching covering all but a very small set of vertices. After absorbing the uncovered vertices by $M$, we obtain a perfect matching in $H$.

\section{Acknowledgements}

This research was partially carried out whilst the authors were visiting the Institute for Mathematics and its Applications at the University of Minnesota. The authors would like to thank the institute for the nice working environment. The authors are also grateful to the referees for their careful reviews.

\section{References}

[1] N. Alon, P. Frankl, H. Huang, V. Rödl, A. Ruciński and B. Sudakov, Large matchings in uniform hypergraphs and the conjectures of Erdös and Samuels, J. Combin. Theory Ser. A 119 (2012), 1200-1215.

[2] A. Czygrinow, V. Kamat, Tight co-degree condition for perfect matchings in 4-graphs, Electron. J. Combin. 19 (2012), no. 2, P20. 
[3] J. Han, Near perfect matchings in $k$-uniform hypergraphs, Combin. Probab. Comput., 24 (2015), 723-732.

[4] J. Han, Perfect Matchings in Hypergraphs and the Erdős matching conjecture, arXiv: 1512.07177.

[5] H. Hàn, Y. Person and M. Schacht, On perfect matchings in uniform hypergraphs with large minimum vertex degree, SIAM J. Discrete Math. 23 (2009), 732-748.

[6] P. Keevash and R. Mycroft, A geometric theory for hypergraph matching, Mem. Amer. Math. Soc. 233 (2014), monograph 1098.

[7] I. Khan, Perfect Matching in 3 uniform hypergraphs with large vertex degree, SIAM J. Discrete Math. 27 (2013), 1021-1039.

[8] I. Khan, Perfect Matchings in 4-uniform hypergraphs, J. Combin. Theory B 116 (2016), 333-366.

[9] D. Kühn and D. Osthus, Matchings in hypergraphs of large minimum degree, $J$. Graph Theory 51 (2006), 269-280.

[10] D. Kühn and D. Osthus, Embedding large subgraphs into dense graphs, in Surveys in Combinatorics (S. Huczynska, J.D. Mitchell and C.M. Roney-Dougal eds.), London Math. Soc. Lecture Notes 365, 137-167, Cambridge University Press, 2009.

[11] D. Kühn, D. Osthus and T. Townsend, Fractional and integer matchings in uniform hypergraphs, European J. Combin. 38 (2014), 83-96.

[12] D. Kühn, D. Osthus and A. Treglown, Matchings in 3-uniform hypergraphs, J. Combin. Theory B 103 (2013) 291-305.

[13] A. Lo and K. Markström, F-factors in hypergraphs via absorption, Graphs Combin. 31 (2015), 679-712.

[14] K. Markström and A. Ruciński, Perfect matchings (and Hamilton cycles) in hypergraphs with large degrees, European J. Combin. 32 (2011), 677-687.

[15] O. Pikhurko, Perfect matchings and $K_{4}^{3}$-tilings in hypergraphs of large codegree, Graphs Combin. 24 (2008), 391-404.

[16] V. Rödl and A. Ruciński, Dirac-type questions for hypergraphs - a survey (or more problems for Endre to solve), An Irregular Mind (Szemerédi is 70), Bolyai Soc. Math. Studies 21 (2010), 1-30.

[17] V. Rödl, A. Ruciński and E. Szemerédi, Perfect matchings in uniform hypergraphs with large minimum degree, European J. Combin. 27 (2006), 1333-1349.

[18] V. Rödl, A. Ruciński and E. Szemerédi, Perfect matchings in large uniform hypergraphs with large minimum collective degree, J. Combin. Theory Ser. A 116 (2009), 613-636.

[19] A. Treglown and Y. Zhao, Exact minimum degree thresholds for perfect matchings in uniform hypergraphs, J. Combin. Theory A 119 (2012), 1500-1522.

[20] A. Treglown and Y. Zhao, Exact minimum degree thresholds for perfect matchings in uniform hypergraphs II, J. Combin. Theory A 120 (2013), 1463-1482. 